\title{
MiR-129-5p sensitization of lung cancer cells to etoposide-induced apoptosis by reducing YWHAB
}

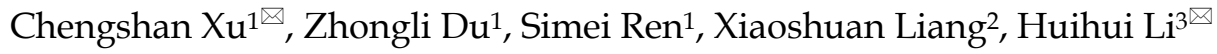 \\ 1. National Center for Clinical Laboratories, Beijing Hospital, National Center of Gerontology; Institute of GeriatricMedicine, Chinese Academy of Medical \\ Sciences, Beijing, China \\ 2. Department of Breast Surgery, Harbin Medical University Cancer Hospital, Harbin, China \\ 3. Department of Medical Oncology, Shandong Cancer Hospital and Institute, Shandong First Medical University and Shandong Academy of Medical \\ Sciences, Jinan, Shandong Province, China \\ $\square$ Corresponding author: E-mail address: Chengshan Xu (chshxu83@163.com); Huihui Li (15553103209@163.com)
}

(C) The author(s). This is an open access article distributed under the terms of the Creative Commons Attribution License (https://creativecommons.org/licenses/by/4.0/). See http://ivyspring.com/terms for full terms and conditions.

Received: 2019.04.02; Accepted: 2019.09.22; Published: 2020.01.01

\begin{abstract}
Background: Lung cancer is the most common cause of death from cancer worldwide and recent studies have revealed that microRNAs play critical roles to regulate lung carcinogenesis. microRNA-129-5p (miR-129-5p) has been reported to regulate cell proliferation and invasion in lung cancer, but its role in lung cancer apoptosis remains unknown.

Methods: The expression of miR-129-5p and YWHAB in lung cancer tissues were analyzed from data downloaded from the NCBI Gene Expression Omnibus (GEO) database. Luciferase reporter assay, Western blot and qRT-PCR were used to determine the regulatory effect of miR-129-5p on YWHAB. Cell apoptosis was detected by using the PI/Annexin V Cell Apoptosis Kit. The effect of miR-129-5p and YWHAB on the survival of lung cancer patients was also explored.

Results: In this study, by combining the data derived from six GEO database, our results showed that miR-129-5p was downregulated in lung cancer tissues and YWHAB was upregulated in lung cancer patient' serum. A significant negative correlation between miR-129-5p and YWHAB was found in lung cancer tissues. Both the expression of YWHAB and miR-129-5p were associated significantly with prognosis (overall survival) in patients with lung cancer. Overexpression of miR-129-5p promotes VP16-induced lung cancer cell apoptosis and YWHAB was shown to be a direct downstream target of miR-129-5p.
\end{abstract}

Conclusion: Overexpression of expression miR-129-5p contributes to etoposide-induced lung cancer apoptosis by modulating YWHAB.

Key words: Lung cancer; miR-129-5p; YWHAB; Apoptosis

\section{Introduction}

Lung cancer is the leading cause of cancerrelated death worldwide, accounting for more than 1.8 million new cases and almost 1.6 million estimated deaths in 2012 [1]. In China, lung cancer is the most commonly diagnosed cancer and the leading cause of cancer-related death, which cause about 6 million deaths in 2015 [2]. Lung cancers are broadly classified into two types: non-small-cell lung cancer (NSCLC) and small-cell lung cancer; NSCLC accounts for the majority of all lung cancer cases, which comprise three major histological subtypes: adenocarcinoma, squamous cell carcinoma and large-cell carcinoma [3]. microRNAs (miRNAs), a kind of small endogenous noncoding RNAs, have been clearly demonstrated to suppress target messenger RNA (mRNA) expression post-transcriptionally by base-pairing with $3^{\prime}$-untranslated region (3'-UTR) of their targets. Differential expression of miRNAs has been revealed between lung cancer and normal tissues and is involved in the progression of lung 
cancer [4-6].

Aberrant expression of miR-129 has been detected in various types of human cancers and the validated target genes are involved in cancer-related biological processes such as cell proliferation, apoptosis, cell cycle, and metastasis [7-11]. Accumulating evidence indicates that miR-129 could play a dual role in tumorigenesis. miR-129 plays a role as tumor suppressors with decreased expression in various tumors [12-16]. However, the role of miR-129 in tumorigenesis remains largely elusive [17].

YWHAB encodes a number of 14-3-3 family proteins, which regulates signal transduction by binding to specific Ser/Thr-phosphorylated motifs containing proteins. 14-3-3 $\beta$, which is encoded by the YWHAB, regulates multiple signaling pathways in normal and cancer cells. 14-3-3 $\beta$ suppressed Cyclin D1 expression and repressed the apoptotic activity through binding phosphorylated SRPK2 and inhibiting its nuclear translocation [18]. 14-3-3 $\beta$ decreased bone formation and inhibits osteoblastogenesis in mesenchymal stem cells trough interact with Ror2 [19]. Sirt2 deacetylated and down-regulated the transcriptional activity of p53, and 14-3-3 $\beta$ augmented the function of Sirt2 in an AKT-dependent manner [20]. 14-3-3 $\beta$ increased glucocorticoid receptor transcriptional activity and hepatic gluconeogenesis [21]. Down regulation of 14-3-3 $\beta$ decreased p-ERK levels and induced senescence phenotypes in glioblastoma cells [22]. Knockdown of 14-3-3 $\beta$ decreased cell viability but increased the LDH release in human glioma U87 cells through inducing ER stress [23]. 14-3-3 $\beta$ was upregualted in hepatocellular carcinoma and enhanced liver cancer cell migration and proliferation $[24,25]$.

\section{Materials and methods}

\section{Cell culture}

A549 and NCI-H1299 (H1299) cell lines purchased from the National Platform of Experimental Cell Resources for Sci-Tech (Beijing, China). Both A549 and H1299 cells were cultured in Dulbecco's modified Eagle's medium with 10\% fetal bovine serum, $100 \mathrm{IU} / \mathrm{mL}$ penicillin, and $100 \mu \mathrm{g} / \mathrm{mL}$ streptomycin at $37^{\circ} \mathrm{C}$ in a humidified $5 \% \quad \mathrm{CO}_{2}$ atmosphere. The medium was changed at alternate days and the cells were split before they reached $100 \%$ confluency.

\section{miRNA mimics, inhibitors, plasmids and cell transfections}

miR-129-5p mimics and miR-129-5p inhibitors were synthesized by Genepharma group (Shanghai,
China). The full-length 3'UTR of YWHAB was subcloned into the pIS0 luciferase plasmid to generate pIS0-YWHAB-3'UTR [26]. Mutant construct of YWHAB-3'UTR, named pIS0-YWHAB-3'UTR-m, which carried a substitution of three nucleotides within the core binding sites of YWHAB-3'UTR, was conducted using mutant PCR primers. Primers used in this study are shown in Supplementary Table 1. Lipofectamine 2000 (Life Technologies Corporation, Grand Island, NY, USA) was used for transfection of DNA plasmids and oligonucleotides according to the manufacturer's protocol.

\section{Quantitative real time-PCR (qRT-PCR)}

We used TRIzol reagent (Life Technologies Corporation) to extract total RNA from the cultured cells. Total RNA was used to synthesize cDNA with a cDNA Synthesis Kit (FastQuant RT Kit, TianGen, Beijing, China). qRT-PCR was performed using SuperReal PreMix Plus (TianGen) according to the manufacturers' recommendations. The sequences of all primers used for qRT-PCR are listed in Supplementary Table 1. All experiments were performed in triplicate. $\beta$-actin and U6 expression were used for normalization of the expression of mRNA and MiR-129-5p, respectively. Relative expression levels were determined using the $2-\Delta \Delta \mathrm{Ct}$ method.

\section{Western blotting}

Total proteins were extracted using lysis buffer (50 mM Tris- $\mathrm{HCl}$ (pH 7.4), $150 \mathrm{mM} \mathrm{NaCl}, 1 \%$ Nonidet P-40, 0.5\% sodium deoxycholate, $50 \mathrm{mM} \mathrm{NaF}, 0.01$ $\mathrm{mM} \mathrm{Na} \mathrm{VO}_{4}$ ) containing phosphatase and protease inhibitors (11873580001, Roche Applied Science). Proteins were separated by sodium dodecyl sulfate-polyacrylamide gel electrophoresis (SDSPAGE) and then transferred onto polyvinylidene fluoride membranes (Millipore, Bedford, MA, USA). After blocked with $5 \%$ bovine serum albumin, the membranes were incubated with the appropriate antibody overnight at $4^{\circ} \mathrm{C}$. The antibodies against YWHAB (Abcam, Cambridge, MA, USA, ab97273) and GAPDH (Abcam, ab8245) were respectively used to detect their targeting proteins. The density of the bands was analyzed by using Image J software (NIH Image, Bethesda, MD) and normalized by GAPDH as indicated.

\section{Luciferase reporter assay}

A549 and H1299 cells were cotransfected with pIS0-YWHAB-3'UTR or pIS0-YWHAB-3'UTR-m and miR-129-5p mimics, $48 \mathrm{~h}$ after transfection, firefly and Renilla luciferase activities were measured according to the Dual-Luciferase Reporter Assay protocol (Promega, Madison, WI, USA). The Renilla luciferase 
activities were used to normalize transfection efficiency. Experiments were performed in triplicate.

\section{Flow cytometry}

Cells transfected with negative control or miR-129-5p mimics/inhibtors for $24 \mathrm{~h}$ were starved overnight and then treated with VP16 (Sigma-Aldrich) for $48 \mathrm{~h}$. The FCM assay was performed using the Annexin V FITC Apoptosis Detection Kit (BD Biosciences, San Jose, CA, USA) according to the manufacturer's instructions.

\section{Statistical analysis}

Data are expressed as the mean \pm standard deviation (SD). Data were analyzed by Student's t-test or analysis of variance using SPSS v.17.0 software (SPSS Inc., Chicago, IL, USA). The statistical significance is defined as $\mathrm{P}<0.05$ (two-sided).
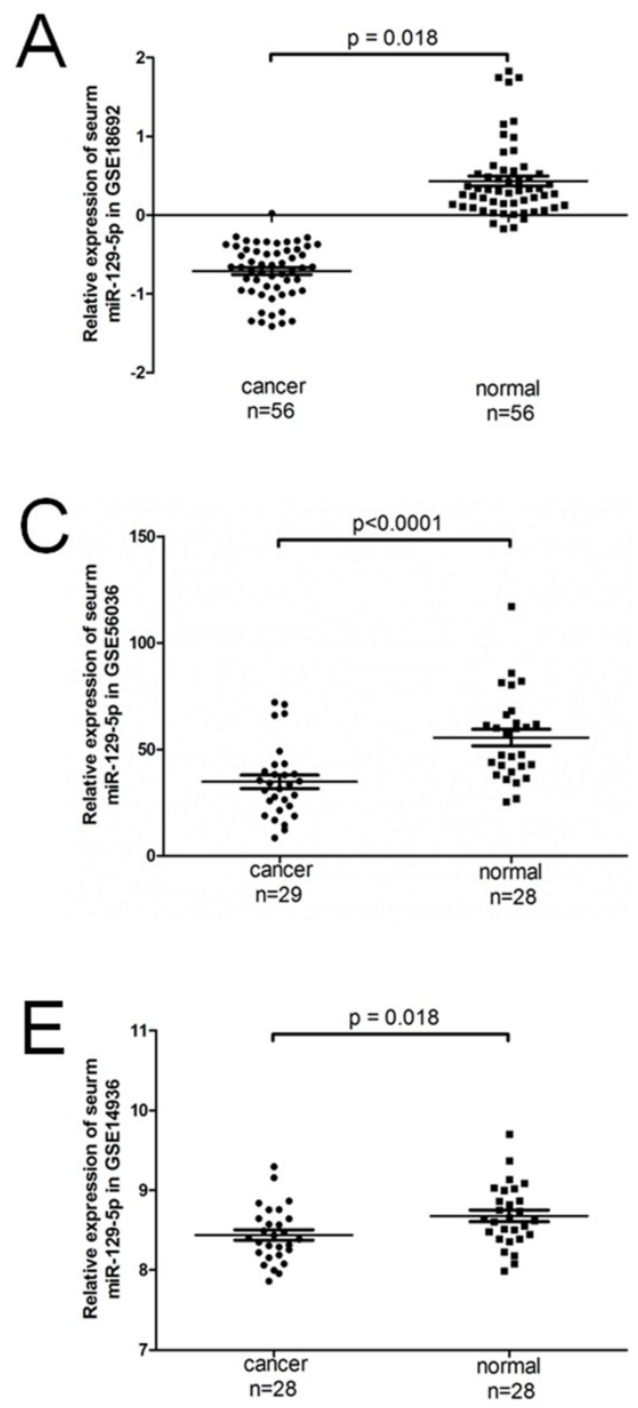

\section{Results}

miR-129-5p is down-regulated in human lung cancer tissues and associated with lung cancer progression

A total of 5 series (GSE) in the GEO database were extracted for the present study, which consisted of 320 lung cancer samples and 319 normal controls. The expression level of miR-129-5p was significantly downregulated in patients with lung cancer in all the 5 GEO datasets (Figure 1A-1E). Compared with the non-cancerous controls, the miR-129-5p lung cancer expression profiles were significantly downregulated in GSE18692, GSE36681, GSE56036, GSE18805 and GSE14936 $(\mathrm{P}<0.05)$. A significant correlation was found between the level of miR-129-5p and over survival of the patients with lung cancer (Figure 1F).
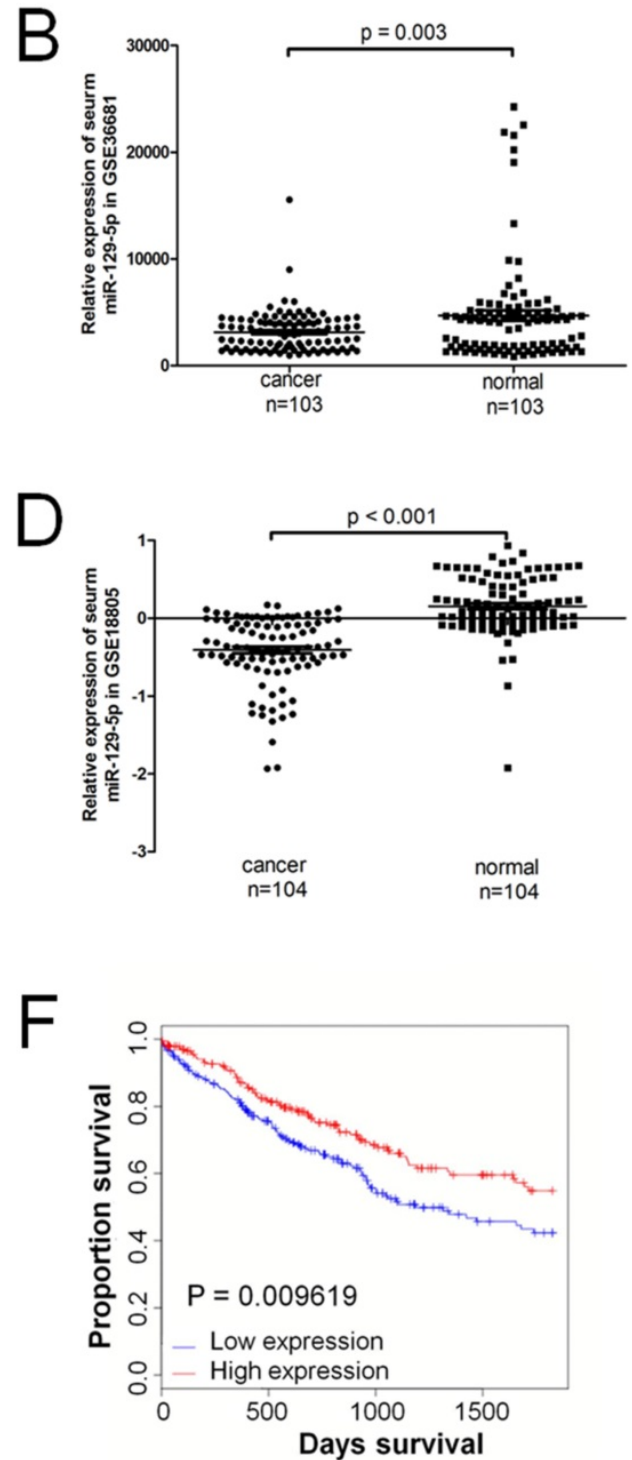

Figure 1. miR-129-5p is down-regulated in human lung cancer tissues and associated with lung cancer progression. Note: (A-E) Relative expression of miR-129-5p in lung cancer tissues and normal control tissues based on the array data retrieved from the GEO online database. (F) Kaplan-Meier survival analysis for the relationship between survival time and miR-129-5p signature in lung cancer was performed by using the online tool (http://kmplot.com/analysis/). 
A
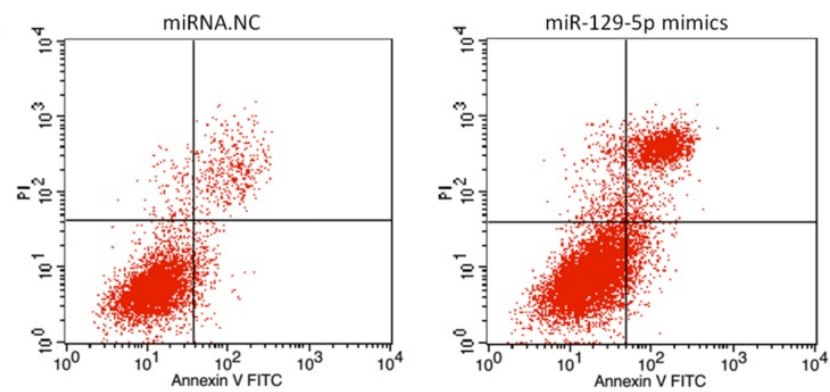

A549 cells

B
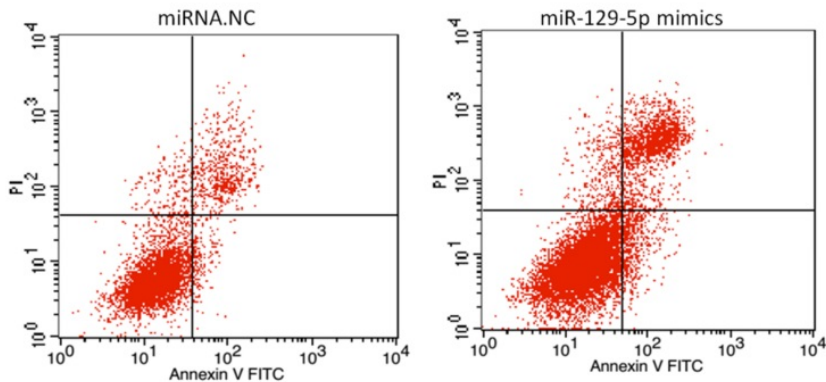

C

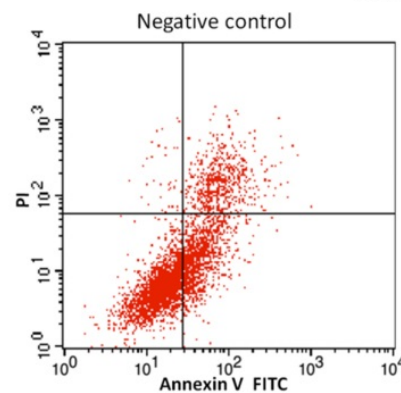

$\mathrm{NCl}-\mathrm{H} 1299$ cells

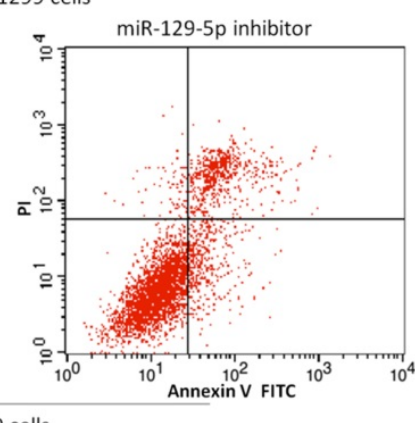

D

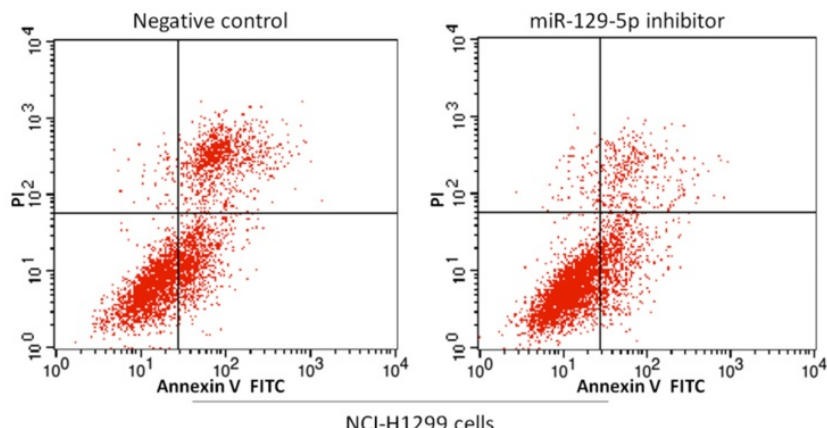

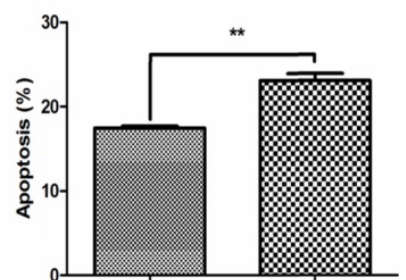

miRNA.NC

$\infty$ miR-129-5p
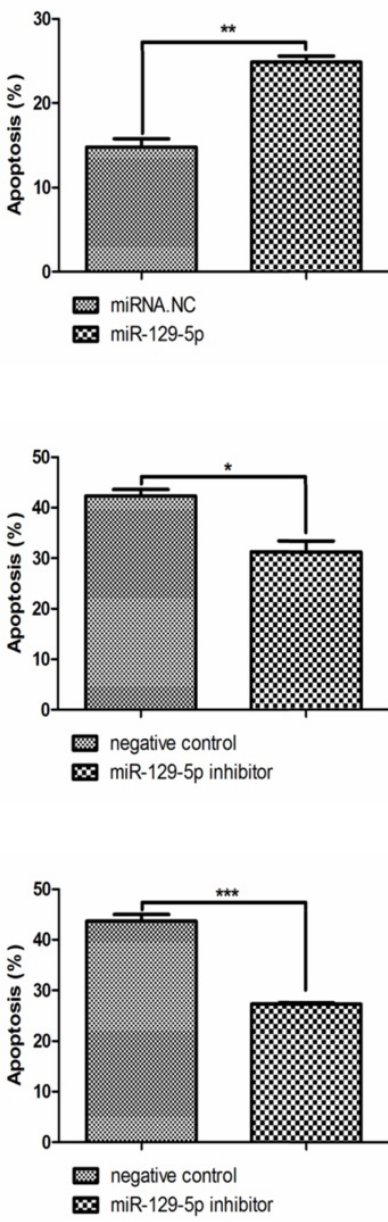

Figure 2. Overexpression of miR-129-5p promotes VP16-induced lung cancer cell apoptosis. Note: (A) Annexin-V FITC/PI assay was used to detect apoptotic cells in A549 cells treated with miR-129-5p mimic/NC (20nM). The rates of apoptosis in A549 cells were quantified (right panel). (B) Annexin-V FITC/PI assay was used to detect apoptotic cells in H1299 cells treated with miR-129-5p mimic/NC (20nM). The rates of apoptosis in H1299 cells were quantified (right panel). (C) Annexin-V FITC/PI assay was used to detect apoptotic cells in A549 cells treated with miR-129-5p inhibitor/NC (40nM). The rates of apoptosis in A549 cells were quantified (right panel). (D) Annexin-V FITC/PI assay was used to detect apoptotic cells in H1299 cells treated with miR-129-5p inhibitor/NC (40nM). The rates of apoptosis in H1299 cells were quantified (right panel).

\section{Overexpression of miR-129-5p promotes VP16-induced lung cancer cell apoptosis}

To verify the effects of miR-129-5p on lung cancer cell apoptosis, we transfected A549 and H1299 cells with miR-129-5p mimics or miR-129-5p inhibitors, followed by the treatment of VP16 for $48 \mathrm{~h}$. FCM was performed to detect the number of apoptotic cells including early and late apoptosis. The results indicated that overexpression of the miR-129-5p mimic markedly promoted VP16 induced apoptosis (Figure 2A and 2B). By contrast, overexpression of miR-129-5p inhibitor elicited the opposite effect, inhibiting VP16-induced apoptosis (Figure 2C and 2D). These results showed that overexpression of miR-129-5p increased the apoptosis of human lung cancer cells induced by the VP16 treatment. 


\section{YWHAB is a direct downstream target of miR-129-5p}

To reveal underlying mechanism by which miR-129-5p regulates cell apoptosis in lung cancer, we next searched for potential target genes of miR-129-5p in TargetScan (targetscan.org). The searching results
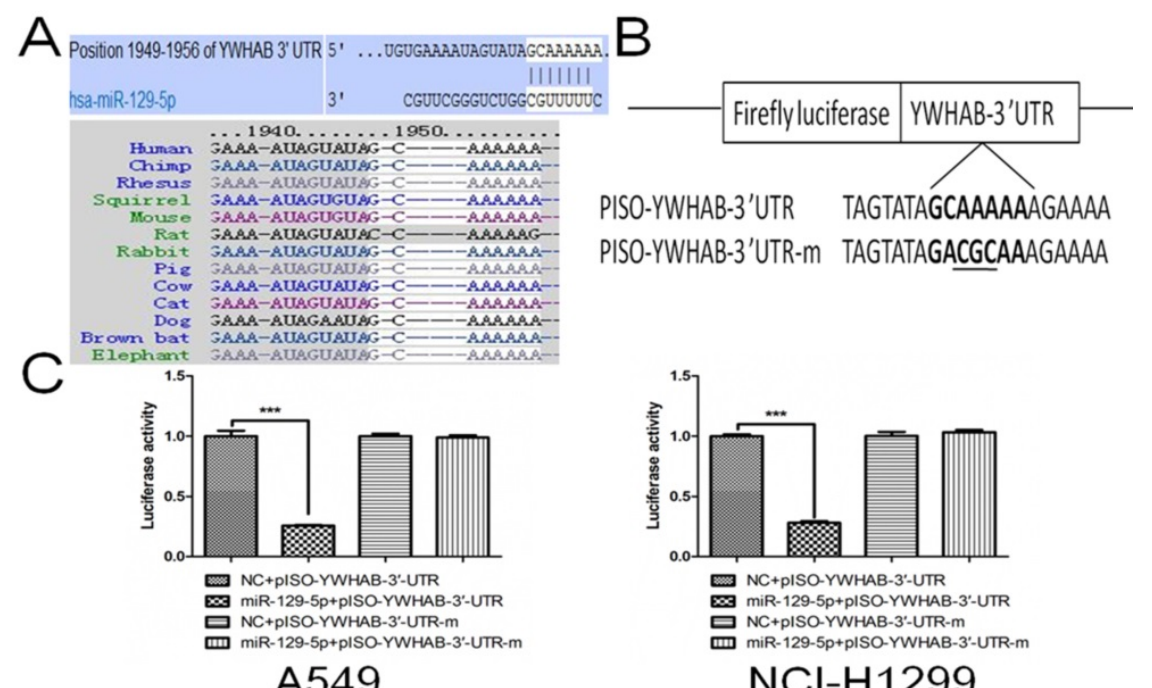

PISO-YWHAB-3'UTR TAGTATAGCAAAAAAGAAAA PISO-YWHAB-3'UTR-m TAGTATAGACGCAAAGAAAA
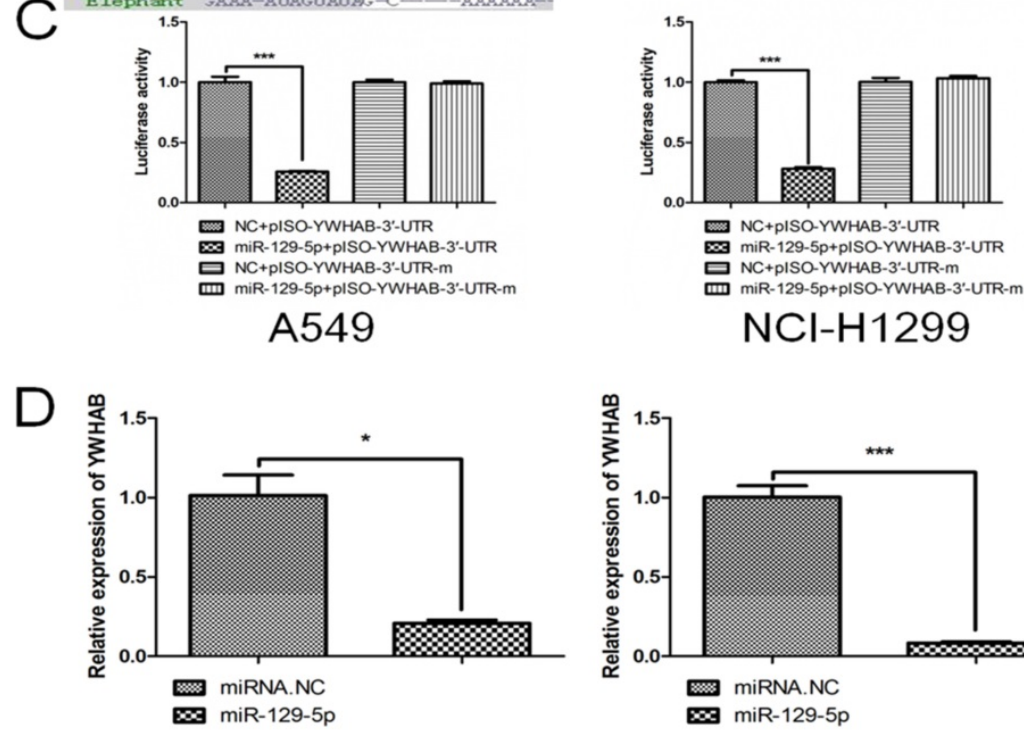

A549
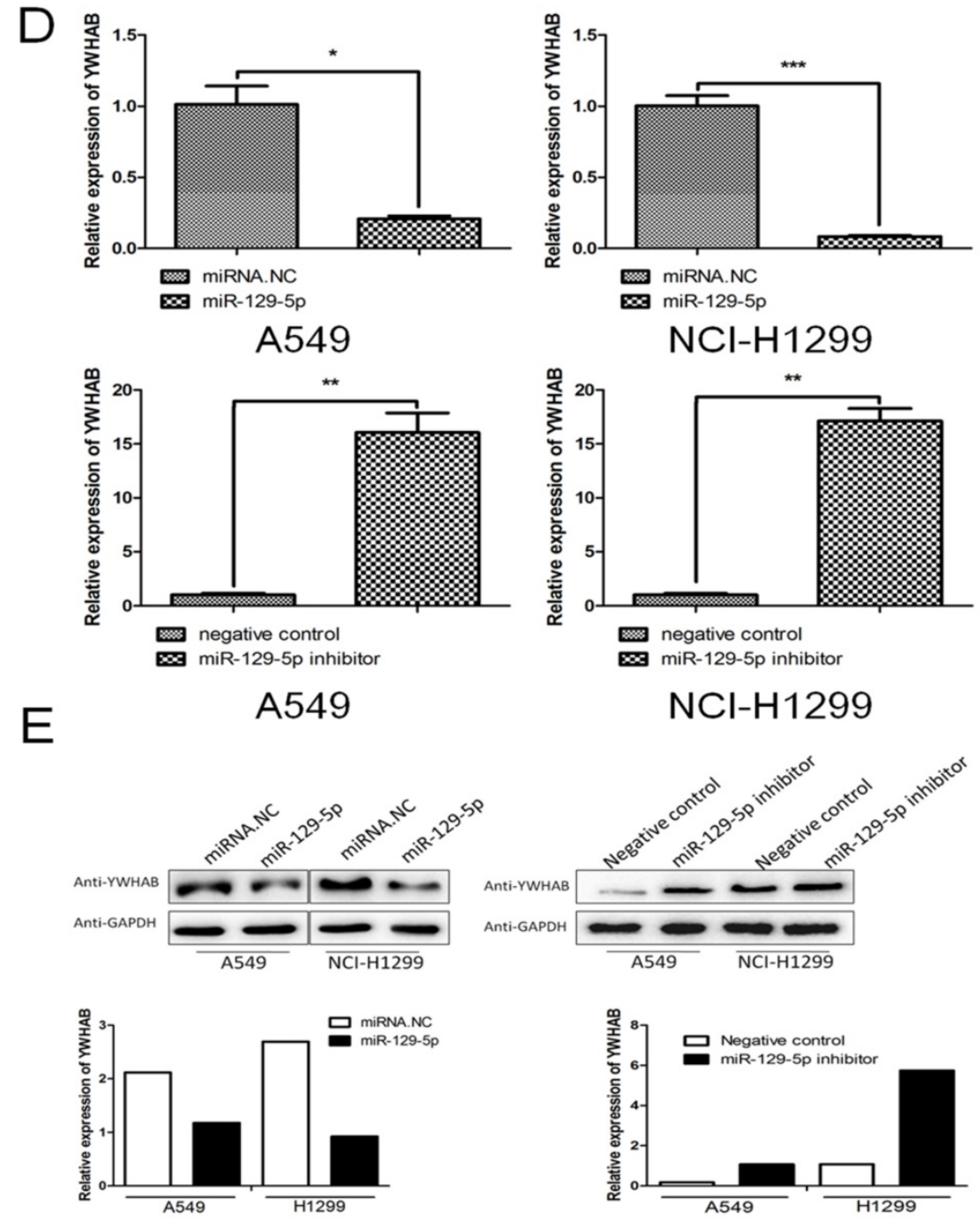

predicted YWHAB as a candidate target of miR-129-5p. The 3'UTR of the YWHAB mRNA contained a complementary site for the seed region of miR-129-5p (Figure 3A upper panel). Homology search showed that the miR-129-5p targeting sequence at nucleotides 1949-1956 of the YWHAB-3'UTR was highly conserved among 13 species (Figure 3A bottom panel). To further validate whether miR-129-5p would bind directly to the $3^{\prime}$ UTR of YWHAB, luciferase reporter vectors containing wide type (WT) 3'UTR or mutant 3'UTR target sequences were constructed. These constructs were cotransfected into A549 and H1299 cells with miR-129-5p mimic or its negative control for performing luciferase reporter assay (Figure 3B). Results demonstrated that miR-129-5p could repress the expression of reported gene containing WT 3 'UTR but not the mutant 3'UTR (Figure 3B). The mRNA and protein levels of YWHAB in A549 and H1299 cells were also determined. The results showed that miR-129-5p reduced YWHAB expression at the mRNA and protein levels (Figure $3 \mathrm{D}$ and 3E). Collectively, these findings suggest that YWHAB is a direct downstream target of miR-129-5p.

Figure 3. YWHAB is a direct downstream target of miR-129-5p. Note: (A) The potential interaction between miR-129-5p and putative binding sites in the YWHAB 3'-UTR predicted by TargetScan (upper panel). A highly-conserved miR-129-5p targeting sequence was predicted in the 3'-UTR of the YWHAB mRNA (bottom panel). (B) The sequences of the mutated YWHAB 3' UTR, in which several nucleotides within the miR-129-5p binding site were mutated. (C) The luciferase activity in the A549 and H1299 cells. Cells were cotransfected with miRNAs and luciferase report plasmids and the luciferase activities were detected in different groups. Each value is evaluated by the relative luciferase activity of firefly to renilla. (D) Effect of miR-129-5p on YWHAB mRNA level in the A549 and H1299 cells. After the cells were transfected, the YWHAB protein expression was detected by RT-PCR. $\beta$-actin was used for the internal control. (E) Effect of miR-129-5p on YWHAB protein level in the A549 and H1299 cells. After the cells were transfected, the YWHAB protein expression was detected by Western blot. GAPDH was used for the internal control. Relative levels of YWHAB were analyzed by quantification of the density of the bands with Image J software (bottom panel). 
YWHAB is the key mediator of the effects of miR-129-5p in lung cancer cells

To confirm that YWHAB is a functional target of miR-129-5p, A549 and H1299 cells were cotransfected with a negative control miRNA (NC) or miR-129-5p mimic, or a plasmid expressing YWHAB. As shown in Figure 4A, fewer apoptotic cells were detected after cotransfection with pCDNA3-YWHAB and miR-129-5p mimics compared to miR-129-5p alone. The same phenomenon was also observed in H1299 cells by FCM (Figure 4B). These results were consistent with the effects of miR-129-5p overexpression, indicating that miR-129-5p regulates lung cells apoptosis by directly targeting YWHAB.
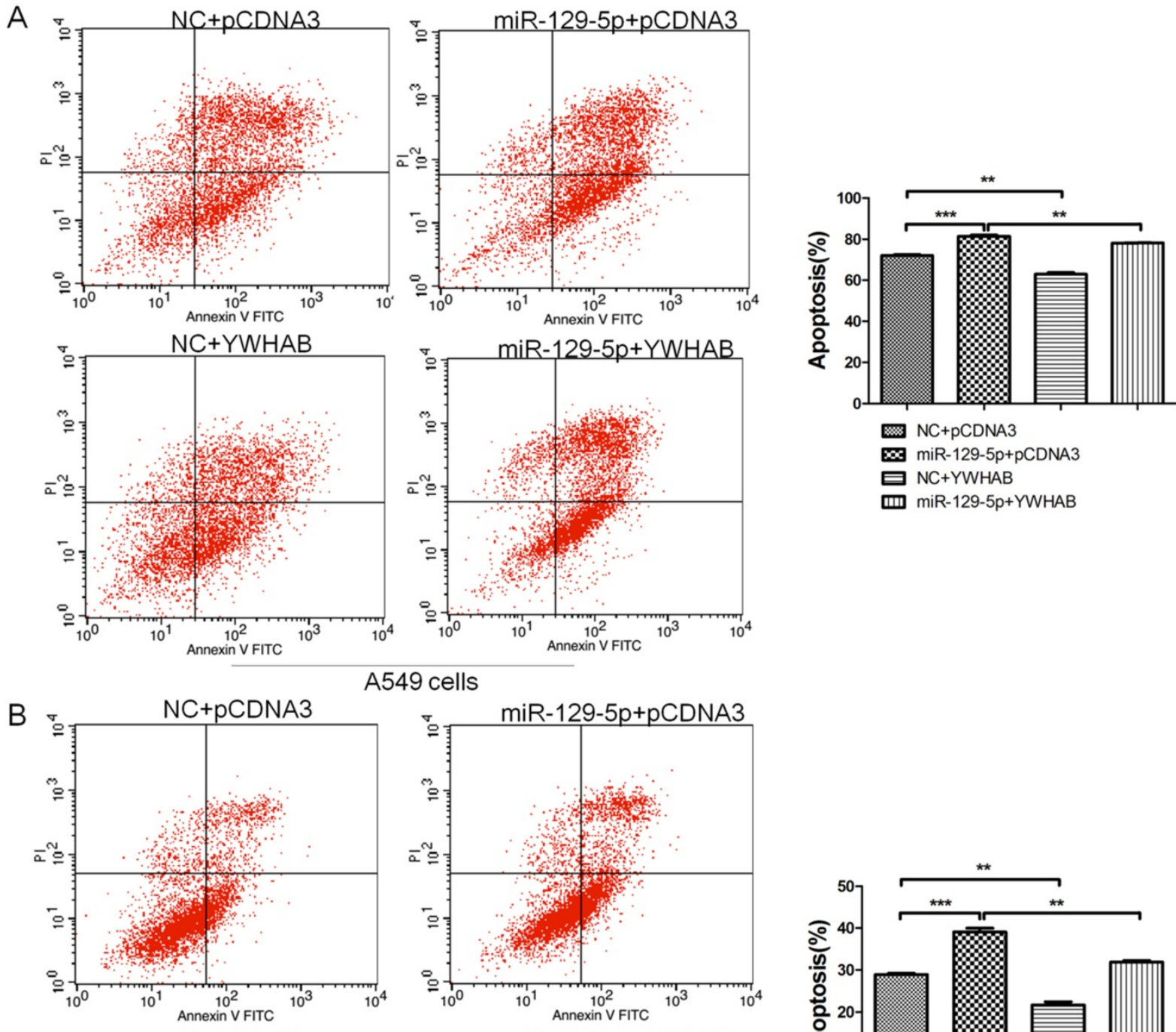

A549 cells
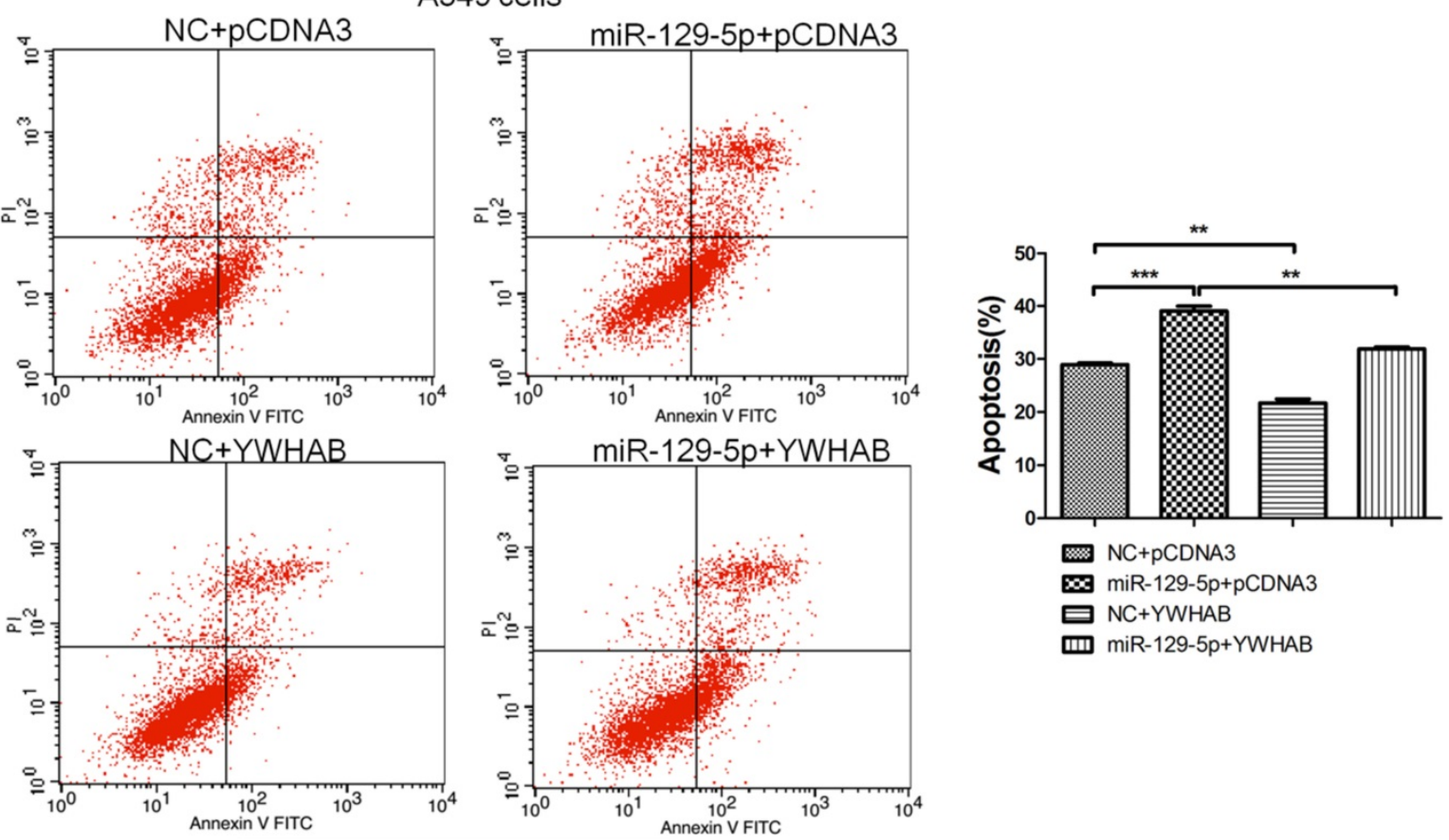

$\mathrm{NCl}-\mathrm{H} 1299$ cells

Figure 4. YWHAB is the key mediator of the effects of miR-129-5p in lung cancer cells. Note: (A) A549 cells were transfected with negative control (NC), miR-129-5p mimics and/or PCDNA3-YWHAB, and then cells were analyzed for apoptotic rate after staining with Annexin V-FITC and PI. Data represent means \pm S.D. from three independent experiments, ** $\mathrm{P}<0.01$; *** $\mathrm{P}<0.001$. (B) $\mathrm{H} 1299$ cells were transfected with negative control (NC), miR-129-5p mimics and/or PCDNA3-YWHAB, and then cells were analyzed for apoptotic rate after staining with Annexin V-FITC and PI. Data represent means \pm S.D. from three independent experiments, $* * \mathrm{P}<0.01$; *** $\mathrm{P}<0.001$. 

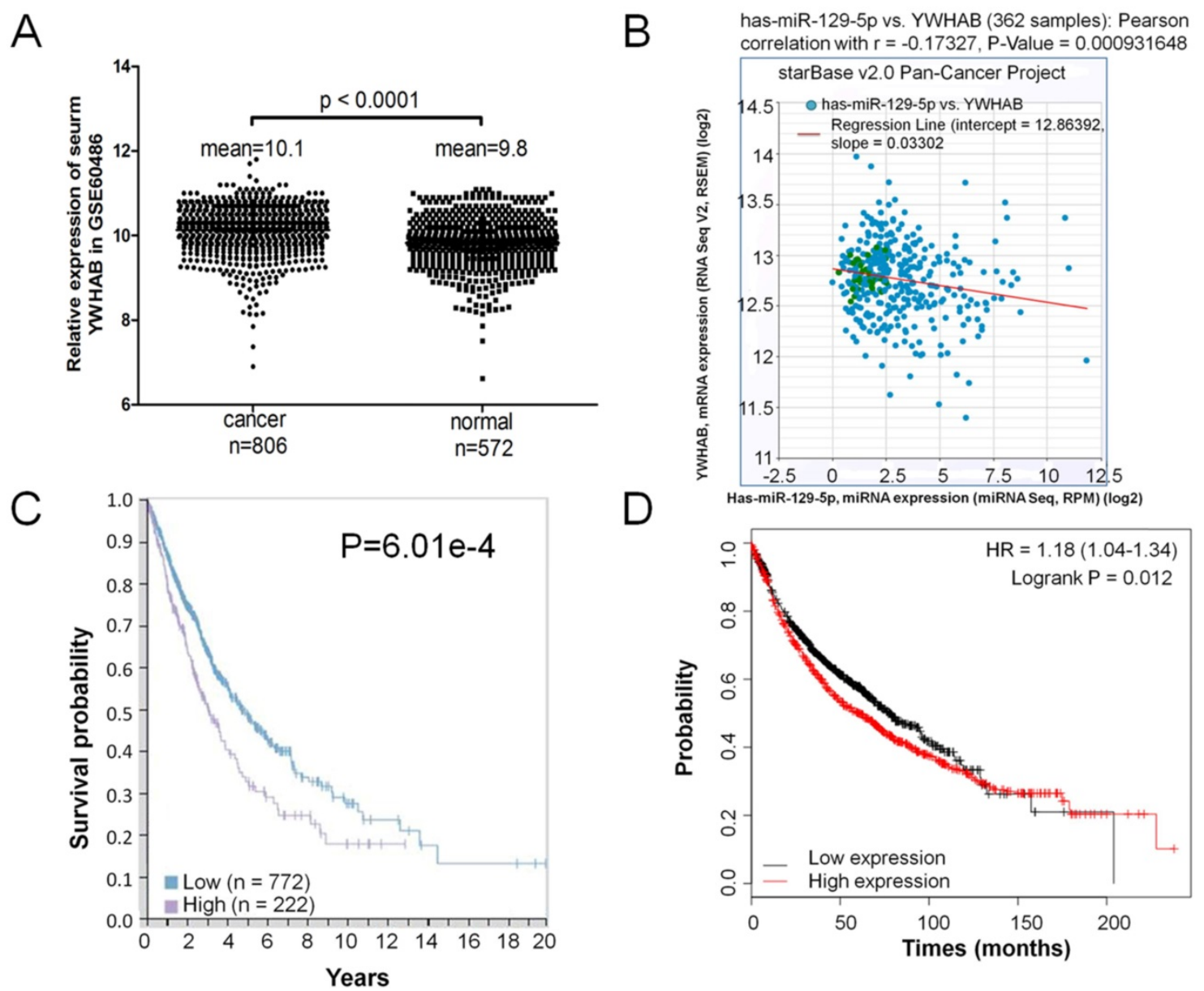

Figure 5. Elevated expression of YWHAB in lung cancer. Note: (A) RT-PCR analysis of GSE60486, YWHAB mRNA levels in patients' serum with lung cancer specimens $(n=806)$ or normal control $(n=572)$. (B) Analysis of correlation between YWHAB and miR-129-5p in lung cancer by using the online tool (http://starbase.sysu.edu.cn/index.php). (C) Kaplan-Meier survival analysis for the relationship between survival time and YWHAB signature in lung cancer was performed by using the online tool (www.proteinatlas.org/pathology). (D) Kaplan-Meier survival analysis for the relationship between survival time and YWHAB signature in lung cancer was performed by using the online tool (http://kmplot.com/analysis/).

\section{Elevated expression of YWHAB in lung cancer}

A GEO database (GSE60486) was extracted for the present study, which consisted of 806 lung cancer samples and 572 normal controls. The expression level of YWHAB was significantly upregulated in patients' serum with lung cancer compared the normal control (Figure 5A). The correlation of miR-129-5p and YWHAB in lung cancer tissues was also determined. With the log10-transformed data, the Pearson correlation analysis showed a significant negative correlation between miR-129-5p and YWHAB $(\mathrm{R}=-0.173, \mathrm{p}=0.0009)$ (Figure 5B). We then explored correlation analyses based on mRNA expression levels of YWHAB in lung cancer tissue and the clinical outcome (survival) of the patient using a recently published interactive open-access database (www.proteinatlas.org/pathology). As expected, increased expression of YWHAB is associated significantly with an unfavorable prognosis (survival) in patients with lung cancer (Figure 5C). We also performed Kaplan-Meier survival analysis of
YWHAB with an online tool (http://kmplot.com/ analysis/). The results showed that higher YWHAB expression was associated with a worse overall survival for patients with lung cancer (Figure 5D).

\section{Discussion}

In this study, five microarray datasets from GEO public database was used to analysis expression of miR-129-5p in lung cancer. Our analysis of publically available databases revealed that miR-129-5p is significantly downregulated in tumor tissue compared with matched adjacent normal tissues. These findings point to an important role for changes in miR-129-5p expression during lung cancer progression.

Although many studies have explored the role of miR-129-5p in cancer [7, 27-29], there are few reports about the relationship between miR-129-5p expression and chemosensitivity of lung cancer. Lu et al. found that miR-129-5p sensitized Her-2-positive breast cancer to trastuzumab by downregulating 
RpS6 [30]. Ma et al. reported that miR-129-5p inhibits NSCLC stemness and chemoresistance through direct targeting of DLK1 [31]. Zeng et al. found that microRNA-129-5p suppresses Adriamycin resistance in breast cancer by targeting SOX2 [32]. These results suggested that miR-129-5p expression may have effects on the chemosensitivity of tumors. In the current study, we examined the effects of miR-129-5p expression on chemosensitivity of A549 cells and H1299 cells. The FCM assay showed that overexpression of miR-129-5p promoted the chemosensitivity of lung cancer cells. Moreover, decreased miR-129-5p level inhibited apoptosis of lung cancer cells after VP16 induction.

In conclusion, our results revealed that miR-129-5p is down-regulated in human lung cancer tissues and associated with lung cancer progression. Overexpression of miR-129-5 promotes VP16induced lung cancer cell apoptosis. YWHAB is a direct downstream target of miR-129-5p and a key mediator of the effects of miR-129-5p in lung cancer cells. Higher YWHAB expression was associated with a worse overall survival for patients with lung cancer. Our findings provide a new role of miR-129-5p in lung cancer, and it may be considered as a potential novel target for therapeutic interventions in lung cancer. miR-129-5p maybe function as a tumor-suppressor in lung cancer and we could deliver it or over-express it in lung cancer cells to enhance lung cancer cell apoptosis. Ectopic expression of miR-129-5p and YWHAB were found in lung cancer tissue or patients' serum and both of them were closely related to the overall survival of patients, which could be used as biomarkers for monitoring the prognosis of lung cancer patients.

\section{Supplementary Material}

Table S1. http://www.jcancer.org/v11p0858s1.pdf

\section{Abbreviations}

GEO: Gene Expression Omnibus; YWHAB: 14-3-3 protein beta/alpha; NSCLC: non-small-cell lung cancer; UTR: Untranslated region; FCM: Flow cytometry; NC: Negative control.

\section{Acknowledgement}

This work was funded by the National Natural Science Foundation of China No. 81501995.

\section{Competing Interests}

The authors have declared that no competing interest exists.

\section{References}

1. Torre LA, Bray F, Siegel RL, Ferlay J, Lortet-Tieulent J, Jemal A. Global cancer statistics, 2012. CA Cancer J Clin. 2015; 65: 87-108.

2. Chen W, Zheng R, Baade PD, Zhang S, Zeng H, Bray F, et al. Cancer statistics in China, 2015. CA Cancer J Clin. 2016; 66: 115-32.

3. Zappa C, Mousa SA. Non-small cell lung cancer: current treatment and future advances. Transl Lung Cancer Res. 2016; 5: 288-300.

4. Garofalo M, Quintavalle C, Di Leva G, Zanca C, Romano G, Taccioli C, et al. MicroRNA signatures of TRAIL resistance in human non-small cell lung cancer. Oncogene. 2008; 27: 3845-55.

5. Volinia S, Calin GA, Liu CG, Ambs S, Cimmino A, Petrocca F, et al. A microRNA expression signature of human solid tumors defines cancer gene targets. Proc Natl Acad Sci U S A. 2006; 103: 2257-61.

6. Yanaihara N, Caplen N, Bowman E, Seike M, Kumamoto K, Yi M, et al. Unique microRNA molecular profiles in lung cancer diagnosis and prognosis. Cancer Cell. 2006; 9: 189-98.

7. Duan L, Hao X, Liu Z, Zhang Y, Zhang G. MiR-129-5p is down-regulated and involved in the growth, apoptosis and migration of medullary thyroid carcinoma cells through targeting RET. FEBS Lett. 2014; 588: 1644-51.

8. Shen Q, Jiang Y. LncRNA NNT-AS1 promotes the proliferation, and invasion of lung cancer cells via regulating miR-129-5p expression. Biomed Pharmacother. 2018; 105: 176-81.

9. Yu X, Song H, Xia T, Han S, Xiao B, Luo L, et al. Growth inhibitory effects of three miR-129 family members on gastric cancer. Gene. 2013; 532: 87-93.

10. Zeng A, Yin J, Li Y, Li R, Wang Z, Zhou X, et al. miR-129-5p targets Wnt5a to block PKC/ERK/NF-kappaB and JNK pathways in glioblastoma. Cell Death Dis. 2018; 9: 394

11. Zhang P, Li J, Song Y, Wang X. MiR-129-5p Inhibits Proliferation and Invasion of Chondrosarcoma Cells by Regulating SOX4/Wnt/beta-Catenin Signaling Pathway. Cell Physiol Biochem. 2017; 42: 242-53.

12. Dyrskjot L, Ostenfeld MS, Bramsen JB, Silahtaroglu AN, Lamy P, Ramanathan $\mathrm{R}$, et al. Genomic profiling of microRNAs in bladder cancer: miR-129 is associated with poor outcome and promotes cell death in vitro. Cancer Res. 2009; 69: 4851-60.

13. Karaayvaz M, Zhai H, Ju J. miR-129 promotes apoptosis and enhances chemosensitivity to 5-fluorouracil in colorectal cancer. Cell Death Dis. 2013; 4: e659.

14. Lu CY, Lin KY, Tien MT, Wu CT, Uen YH, Tseng TL. Frequent DNA methylation of MiR-129-2 and its potential clinical implication in hepatocellular carcinoma. Genes Chromosomes Cancer. 2013; 52: 636-43.

15. Yang $Y$, Huang JQ, Zhang X, Shen LF. MiR-129-2 functions as a tumor suppressor in glioma cells by targeting HMGB1 and is down-regulated by DNA methylation. Mol Cell Biochem. 2015; 404: 229-39.

16. Yu Y, Zhao Y, Sun $\mathrm{XH}, \mathrm{Ge} J$, Zhang B, Wang $\mathrm{X}$, et al. Down-regulation of miR-129-5p via the Twist1-Snail feedback loop stimulates the epithelial-mesenchymal transition and is associated with poor prognosis in breast cancer. Oncotarget. 2015; 6: 34423-36.

17. Li M, Tian L, Wang L, Yao H, Zhang J, Lu J, et al. Down-regulation of miR-129-5p inhibits growth and induces apoptosis in laryngeal squamous cell carcinoma by targeting APC. PLoS One. 2013; 8: e77829.

18. Jang SW, Liu X, Fu H, Rees H, Yepes M, Levey A, et al. Interaction of Akt-phosphorylated SRPK2 with 14-3-3 mediates cell cycle and cell death in neurons. J Biol Chem. 2009; 284: 24512-25.

19. Liu Y, Ross JF, Bodine PV, Billiard J. Homodimerization of Ror2 tyrosine kinase receptor induces 14-3-3(beta) phosphorylation and promotes osteoblast differentiation and bone formation. Mol Endocrinol. 2007; 21: 3050-61.

20. Jin YH, Kim YJ, Kim DW, Baek KH, Kang BY, Yeo CY, et al. Sirt2 interacts with 14-3-3 beta/gamma and down-regulates the activity of p53. Biochem Biophys Res Commun. 2008; 368: 690-5.

21. Hwang Y, An HT, Kang M, Ko J. Roles of 14-3-3beta and gamma in regulation of the glucocorticoid receptor transcriptional activation and hepatic gluconeogenesis. Biochem Biophys Res Commun. 2018; 501: 800-6.

22. Seo SB, Lee JJ, Yun HH, Im CN, Kim YS, Ko JH, et al. 14-3-3beta Depletion Drives a Senescence Program in Glioblastoma Cells Through the ERK/SKP2/p27 Pathway. Mol Neurobiol. 2018; 55: 1259-70.

23. Cao L, Lei H, Chang MZ, Liu ZQ, Bie XH. Down-regulation of 14-3-3beta exerts anti-cancer effects through inducing ER stress in human glioma U87 cells: Involvement of CHOP-Wnt pathway. Biochem Biophys Res Commun. 2015; 462: 389-95.

24. Liu TA, Jan YJ, Ko BS, Chen SC, Liang SM, Hung YL, et al. Increased expression of 14-3-3beta promotes tumor progression and predicts extrahepatic metastasis and worse survival in hepatocellular carcinoma. Am J Pathol. 2011; 179: 2698-708.

25. Tang Y, Lv P, Sun Z, Han L, Zhou W. 14-3-3beta Promotes Migration and Invasion of Human Hepatocellular Carcinoma Cells by Modulating Expression of MMP2 and MMP9 through PI3K/Akt/NF-kappaB Pathway. PLoS One. 2016; 11: e0146070.

26. Yekta S, Shih IH, Bartel DP. MicroRNA-directed cleavage of HOXB8 mRNA. Science. 2004; 304: 594-6.

27. Chen D, Wang H, Chen J, Li Z, Li S, Hu Z, et al. MicroRNA-129-5p Regulates Glycolysis and Cell Proliferation by Targeting the Glucose Transporter SLC2A3 in Gastric Cancer Cells. Front Pharmacol. 2018; 9: 502. 
28. Fu L, Chen $\mathrm{Q}$, Yao T, Li T, Ying S, Hu Y, et al. Hsa_circ_0005986 inhibits carcinogenesis by acting as a miR-129-5p sponge and is used as a novel biomarker for hepatocellular carcinoma. Oncotarget. 2017; 8: 43878-88.

29. Lin J, Shi Z, Yu Z, He Z. LncRNA HIF1A-AS2 positively affects the progression and EMT formation of colorectal cancer through regulating miR-129-5p and DNMT3A. Biomed Pharmacother. 2018; 98: 433-9.

30. Lu X, Ma J, Chu J, Shao Q, Zhang Y, Lu G, et al. MiR-129-5p Sensitizes the Response of Her-2 Positive Breast Cancer to Trastuzumab by Reducing Rps6. Cell Physiol Biochem. 2017; 44: 2346-56.

31. Ma Z, Cai H, Zhang Y, Chang L, Cui Y. MiR-129-5p inhibits non-small cell lung cancer cell stemness and chemoresistance through targeting DLK1. Biochem Biophys Res Commun. 2017; 490: 309-16.

32. Zeng $\mathrm{H}$, Wang L, Wang J, Chen T, Li H, Zhang K, et al. microRNA-129-5p suppresses Adriamycin resistance in breast cancer by targeting SOX2. Arch Biochem Biophys. 2018; 651: 52-60. 